\title{
Analysis of facial motor evoked potentials for assessing a central mechanism in hemifacial spasm
}

\author{
Marshall F. Wilkinson, PhD, ${ }^{1}$ Tumul Chowdhury, MD, DM, FRCPC, ${ }^{2}$ W. Alan Mutch, $\mathrm{MD},{ }^{2}$ and \\ Anthony M. Kaufmann, MD, MSc, FRCSC ${ }^{1}$ \\ ${ }^{1}$ Section of Neurosurgery and 2Department of Anesthesiology and Perioperative Medicine, Faculty of Medicine, University of \\ Manitoba and Health Sciences Centre, Winnipeg, Manitoba, Canada
}

\begin{abstract}
OBJECTIVE Hemifacial spasm (HFS) is a cranial nerve hyperactivity disorder characterized by unique neurophysiological features, although the underlying pathophysiology remains disputed. In this study, the authors compared the effects of desflurane on facial motor evoked potentials (MEPs) from the spasm and nonspasm sides of patients who were undergoing microvascular decompression (MVD) surgery to test the hypothesis that HFS is associated with a central elevation of facial motor neuron excitability.
\end{abstract}

METHODS Facial MEPs were elicited in 31 patients who were undergoing MVD for HFS and were administered total intravenous anesthesia (TIVA) with or without additional desflurane, an inhaled anesthetic known to centrally suppress MEPs. All measurements were completed before dural opening while a consistent mean arterial blood pressure was maintained and electroencephalography was performed. The activation threshold voltage and mean amplitudes of the MEPs from both sides of the face were compared.

RESULTS There was a significantly lower mean activation threshold of facial MEPs on the spasm side than on the nonspasm side (mean \pm SD $162.9 \pm 10.1$ vs $198.3 \pm 10.1 \mathrm{~V}$, respectively; $p=0.01$ ). In addition, MEPs were also elicited more readily when single-pulse transcranial electrical stimulation was used on the spasm side $(74 \%$ vs $31 \%$, respectively; $p=0.03$ ). Although desflurane (1 minimum alveolar concentration) suppressed facial MEPs on both sides, the suppressive effects of desflurane were less on the spasm side than on the nonspasm side ( $59 \%$ vs $79 \%$, respectively; $p$ $=0.03$ ), and $\mathrm{M}$ waves recorded from the mentalis muscle remained unchanged, which indicates that desflurane did not affect the peripheral facial nerve or neuromuscular junction.

CONCLUSIONS Centrally acting inhaled anesthetic agents can suppress facial MEPs and therefore might interfere with intraoperative monitoring. The elevated motor neuron excitability and differential effects of desflurane between the spasm and nonspasm sides support a mechanism of central pathophysiology in HFS.

Clinical trial registration no.: B2012:099 (clinicaltrials.gov)

http://thejns.org/doi/abs/10.3171/2016.2.JNS151384

KEY WORDS hemifacial spasm; motor evoked potentials; desflurane; facial motor neurons; microvascular decompression; functional neurosurgery

$\mathrm{H}$ EMIFACIAL spasm (HFS) is typically characterized by unilateral, irregular twitching of the facial muscles and is associated with chronic neurovascular contact near the facial nerve root exit zone. ${ }^{11}$ HFS tends to increase in severity over time and compromises patient quality of life. However, there are 2 highly successful treatment options: serial botulinum toxin injections to the affected facial muscles and microvascular decompression (MVD) to alleviate the culprit neurovascular compression.
Although the etiological features of HFS include chronic neurovascular contact, it is apparent that this blood vessel-nerve juxtaposition establishes subsequent pathophysiological changes that are directly responsible for HFS symptoms. In particular, there are 2 main hypotheses for explaining the pathophysiological changes that arise with HFS. The peripheral hypothesis suggests that axo-axonal (ephaptic) transmission occurs at the vascular compression site, where facial nerve demyelination results in "lateral

ABBREVIATIONS EEG = electroencephalographic; EMG = electromyographic; HFS = hemifacial spasm; LSR = lateral spread response; MAC = minimum alveolar concentration; MEP = motor evoked potential; MVD = microvascular decompression; TIVA = total intravenous anesthesia.

SUBMITTED June 15, 2015. ACCEPTED February 25, 2016.

INCLUDE WHEN CITING Published online May 13, 2016; DOI: 10.3171/2016.2.JNS151384. 
spreading" of orthodromically directed axonal conduction impulses. ${ }^{17,24,25,33,38,39}$ The central hypothesis suggests that excitability within the facial motor neuron pool is a result of chronic antidromic signaling produced by the rhythmic pulsing on facial nerve axons. $5,7,8,10,13,20,29,36$ The study of these 2 hypotheses has led to an interesting scientific debate and clever experimental designs for investigating the nature of the pathophysiology of HFS.

A unique neurophysiological feature of HFS is the lateral spread response (LSR). This electromyographic (EMG) signature of HFS is produced by electrically stimulating 1 branch of the facial nerve and observing an EMG response from muscles innervated by a different facial nerve branch. ${ }^{21-23}$ Thus, the stimulus laterally spreads to include other facial nerve branches and muscles. The LSR has been studied frequently in attempts to understand the pathophysiology of HFS. Our group began investigating the peripheral and central hypotheses by examining facial motor evoked potentials (MEPs) acquired during MVD for HFS. ${ }^{37}$ We have described the reduction or abolition of spasm-side MEPs after MVD that was coincident with the reduction or disappearance of the LSR. This observation has since been reported by other groups. ${ }^{6-8} \mathrm{Al}-$ though MEPs are unquestionably centrally generated, ${ }^{19}$ correlation of MEP changes with the LSR does not definitively identify the locus of action for the LSR or the site of pathophysiology in HFS.

We previously investigated the effects of desflurane on the LSR..$^{35}$ In this study, we sought to examine the effects of desflurane on facial MEPs from both spasm-side and control-side facial muscles in patients who were undergoing MVD surgery for HFS. In particular, we assessed facial MEPs in the patients after administering to them total intravenous anesthesia (TIVA) with or without desflurane, because the locus of action of the inhaled anesthetic is within the central nervous system and is known to be suppressive on other types of MEPs. We hypothesized that if peripheral ephaptic transmission is a cause of HFS, then desflurane should influence facial MEPs equally on spasm- and non-spasm-side muscles. However, a central mechanism for HFS pathophysiology would be expected to manifest with differences in desflurane responses between spasm-side and non-spasm-side facial MEPs.

\section{Methods}

Patients were recruited in this prospective study after they provided signed informed consent. Our protocol was reviewed and approved by our local institutional ethics committee. The study was registered with the clinicaltrials. gov database under registration number B2012:099.

Included in the study were patients with HFS of either sex between 18 and 75 years of age with otherwise normal neurological examination results and an American Society of Anesthesiologists Physical Status Scale ${ }^{30}$ grade of 1 or 2. Exclusion criteria were existing neuromuscular disease, previous surgery with facial nerve involvement, preexisting significant medical disease, clinically detectable facial neuropathy (House-Brackmann Grade ${ }^{9} \geq 2$ ), and receipt of botulinum toxin injection(s) within 6 months before surgery.

\section{Anesthesia Protocol}

Standard monitors were applied and radial artery cannulation was performed before the induction of anesthesia. We induced anesthesia in each patient according to a standard protocol, which included a remifentanil bolus (1 $\mu \mathrm{g} / \mathrm{kg}$ ), a propofol bolus ( $2-3 \mathrm{mg} / \mathrm{kg}$ ), and a depolarizing muscle relaxant $(1.5 \mathrm{mg} / \mathrm{kg}$ succinylcholine), and the trachea was intubated using an appropriately sized flexometallic endotracheal tube. No neuromuscular blockade was used thereafter. The restoration of neuromuscular function was assessed by using the train-of-four method when stimulating the mandibular branch of the facial nerve and recording the mentalis muscle $\mathrm{M}$ wave.

Anesthesia was maintained with the administration of TIVA (propofol [100-300 $\mu \mathrm{g} / \mathrm{kg} / \mathrm{min}]$ and remifentanil [0.05-0.2 $\mu \mathrm{g} / \mathrm{kg} / \mathrm{min}]$ infusions). Total fresh gas flow was maintained at $2 \mathrm{~L} / \mathrm{min}$ with $40 \%$ oxygen in air, and the end-tidal $\mathrm{CO}_{2}$ level was maintained at $30-35 \mathrm{~mm} \mathrm{Hg}$. For the study, recordings were obtained after the administration of TIVA alone and of TIVA plus desflurane; the desflurane was initiated at $8 \%$, and flow was increased to $8 \mathrm{~L} /$ min to rapidly achieve a depth of anesthesia of 0.5 and/or 1.0 minimum alveolar concentration (MAC). At least 10 minutes was allowed for equilibration after the introduction or complete elimination of desflurane, as assessed by end-tidal monitoring. All study measurements were obtained before opening the dura mater. Mean arterial blood pressure was kept at $70-90 \mathrm{~mm} \mathrm{Hg}$ by using intravenous phenylephrine $(100 \mu \mathrm{g}) /$ ephedrine $(5-10 \mathrm{mg})$ boluses as necessary. Normothermia was maintained throughout the procedure. After completion of the study protocol, desflurane was reduced to $\leq 0.5 \mathrm{MAC}$ throughout the procedure.

\section{Neurophysiology}

On the patient's spasm side, facial muscle electromyography was performed with paired subdermal needles (Medtronic) inserted into the orbicularis oculi, orbicularis oris, and mentalis muscles. On the control side, the paired subdermal needles were inserted into the orbicularis oris and mentalis muscles. Impedances were below $5 \mathrm{kHz}$. The signals were amplified, filtered $(30-3000 \mathrm{~Hz}$ ), and displayed with a commercial neuromonitoring workstation (Cadwell Cascade or Cadwell Elite).

Although LSR data were not the focus of this study, stimulation of the mandibular branch of the facial nerve to evoke the LSR from the orbicularis oculi also produced an $\mathrm{M}$ wave from the mentalis muscle, which we used to assess the level of neuromuscular blockade and to determine the effects of desflurane on the neuromuscular junction. This stimulation was achieved via constant current monophasic pulses (Cadwell ES IX) of $200 \mu \mathrm{sec}, 1 \mathrm{~Hz}$, and between 6 and $40 \mathrm{~mA}$ over the mandibular branch of the facial nerve using 2-cm adhesive disc-type electrodes (Cadwell).

The MEPs from the facial muscles were elicited by using transcranial anodal electrical stimulation delivered through subdermal needle electrodes (Ambu) positioned at C-3 (right face) or C-4 (left face) and referenced to $\mathrm{Cz}$ (cathode). Single or multipulse anodal stimuli were delivered by using a $50-\mu$ sec pulse width from a Cadwell TCS-1 or TCS-4 stimulator. The MEP stimulus threshold 
was determined from the HFS and control sides in each patient as the minimum stimulus voltage required to elicit a response of $\geq 30 \mu \mathrm{V}$ in at least $50 \%$ of a minimum of 8 trials. ${ }^{35}$ For further testing, the stimulus strength was increased, if necessary, to a level above threshold at which an at least $80 \%$ response rate was achieved. Responses to transcranial electrical stimulation that had an onset latency of $<10 \mathrm{msec}$ were excluded from analysis, because they were considered contaminated by direct current spread to the extracranial facial nerve. ${ }^{4}$

Two-channel electroencephalographic (EEG) activity was monitored in all patients using subdermal needles (Chalgren) positioned at F-3 and F-4 referenced to CPz. The records were amplified and displayed with a 5-second full sweep and filtered from 1 to $70 \mathrm{~Hz}$. Digital analysis of the records was performed continuously and displayed as a density spectral array (DSA) for quantification of the EEG results over time. The EEG results were categorized into 1 of 3 descriptors during the 2 anesthesia conditions. "Isoelectric" was defined as an absence of EEG activity, "burst suppression" was defined as at least 1 to 5 seconds of suppression followed by bursts of EEG activity, and "alpha/delta" was defined by predominant alpha and delta bands derived from the DSA analysis.

\section{Study Protocol}

We compared facial MEPs obtained after the administration of TIVA with those obtained after the administration of TIVA plus desflurane. Because each patient served as his or her own control, we a priori assigned half of the patients to initially undergo TIVA followed by the addition of desflurane to $1 \mathrm{MAC}$, and the other half of the patients underwent a combination of TIVA plus 1-MAC desflurane followed by TIVA alone. Data were to be collected during the initial exposure and craniectomy but before opening of the dura, because changes in the LSR have been known to occur after the dura is opened (which likely result from the relief of intracranial pressure and decreasing the neurovascular contact ${ }^{12}$ ) and could impact facial MEPs as well. When time permitted, data were also obtained from the patients after the administration of 0.5-MAC desflurane. At least 10 minutes was allowed for equilibration after the introduction of desflurane, and often 15 or more minutes was required for the elimination of desflurane (as assessed by end-tidal monitoring) when the patient was switched to TIVA alone. The amount of propofol was adjusted to maintain EEG readings that were similar under either of the 2 anesthetic conditions. At each anesthetic condition, we assessed stimulus threshold, single-pulse responses, and desflurane suppression. It should be noted that not all patients were tested with single-pulse stimulation or 0.5-MAC desflurane. Because all data required collection before opening the dura, the washout time for desflurane often prevented some data from being acquired.

\section{Statistical Analysis}

Individual MEP peak-to-peak amplitudes (microvolts) and onset latencies (milliseconds) were averaged from 10 consecutive responses for each anesthetic condition (TIVA only vs TIVA plus desflurane [0.5 and/or 1.0 MAC]). If the
MEP was abolished with desflurane, then the amplitude was considered to be zero. However, for onset latency, we excluded any zero responses, because they would render the subsequent average meaningless. For the statistical comparison, we examined the mean MEP amplitudes ( \pm SEM) and onset latencies between the 3 HFS facial muscles and the combined MEP observations. A similar analysis was performed for the control (nonspasm) side.

SigmaPlot 13 (Systat Software Inc.) statistical software was used for analysis of the data. For pairwise comparisons, the Mann-Whitney rank-sum U-test was used for nonparametric data, and a paired t-test was used for normally distributed data. For comparison of the effects of desflurane from pooled MEP amplitudes, we used repeated-measures 1-way ANOVA with Holm-Sidak's method for multiple pairwise comparisons. When data were expressed as rates or proportions, the Fisher exact or z test was used for statistical analysis. A difference was considered statistically significant when $\mathrm{p}$ was $<0.05$.

\section{Results}

Thirty-one patients (20 women, 11 men) were recruited into the study between November 2012 and September 2014. We successfully recorded spasm-side MEPs from at least 1 facial muscle in 26 (84\%) of 31 patients and control-side MEPs from 23 (74\%) of 31 patients after the administration of TIVA ( $p=1.0$, Fisher exact test). The mean ( \pm SEM) MEP amplitudes, at or near threshold, from all facial muscles were $142.3 \pm 20.2 \mu \mathrm{V}$ on the HFS side and $111.4 \pm 18.7 \mu \mathrm{V}$ on the control side (see Fig. $2 ; \mathrm{p}=0.84$, Mann-Whitney rank-sum U-test). The threshold voltage that elicited these responses was significantly lower on the spasm side than on the control side $(162.9 \pm 10.1$ vs 198.3 $\pm 10.1 \mathrm{~V}$, respectively; $\mathrm{p}=0.01$, Mann-Whitney rank-sum U-test). The mean MEP amplitudes according to the individual facial muscles are shown in Table 1.

In addition to threshold stimulus voltages, we compared facial motor neuron excitability between the 2 sides by observing the proportion of patients in whom MEPs could be generated using a single transcranial electrical pulse while maintaining criteria for centrally generated responses. ${ }^{4}$ Motor evoked potentials normally require multipulse stimulus trains to sufficiently depolarize motor

TABLE 1. MEP amplitudes from individual muscles in patients with HFS

\begin{tabular}{cccc}
\hline \multirow{2}{*}{ Side } & \multicolumn{3}{c}{ MEP Amplitudes (mean \pm SEM) } \\
\cline { 2 - 4 } & Orbicularis Oculi & Orbicularis Oris & Mentalis \\
\hline $\mathrm{HFS}(\mu \mathrm{V})$ & $104.7 \pm 18.5$ & $96.3 \pm 14.3$ & $232.9 \pm 48.2$ \\
\hline TIVA & $45.2 \pm 13.3^{*}$ & $46.1 \pm 13.3^{*}$ & $86.6 \pm 24.4^{*}$ \\
\hline $\begin{array}{c}\text { TIVA+1.0-MAC } \\
\text { desflurane }\end{array}$ & & \\
\hline $\begin{array}{c}\text { Control }(\mu \mathrm{V}) \\
\text { TIVA }\end{array}$ & $62.6 \pm 10.6$ & $126.4 \pm 23.2$ \\
\hline $\begin{array}{c}\text { TIVA+1.0-MAC } \\
\text { desflurane }\end{array}$ & $8.5 \pm 8.5 \dagger$ & $25.7 \pm 9.5^{*}$ \\
\hline
\end{tabular}

* $p<0.01$ (Mann-Whitney rank-sum U-test).

$\dagger p<0.05$ (Mann-Whitney rank-sum U-test). 
neurons above the action potential threshold.$^{19}$ However, facial MEPs in patients with HFS may be an exception to this rule. ${ }^{6,36,37}$ In the present study we found that on the HFS side, 17 (74\%) of 23 of those patients tested responded to single-pulse stimulation with centrally derived MEPs compared with 4 (31\%) of 13 patients tested on the control side $(\mathrm{p}=0.03, \mathrm{z}$ test). A representative MEP response is shown in Fig. 1.

We also examined the onset latencies of MEPs for those patients responding with single-pulse transcranial stimulation versus multipulse stimulation from both sides; the results are summarized in Table 2. There was a significant difference between single-pulse $(13.1 \pm 0.3 \mathrm{msec}$, all muscles) and multipulse (14.6 $\pm 0.5 \mathrm{msec}$, all muscles) latencies on the HFS side $(\mathrm{p}<0.03)$, although there were no other differences between the HFS- and control-side MEP onset latencies. All MEPs elicited with a single pulse met the centrally derived criteria.

The effects of desflurane on facial MEP responses were compared between the HFS and control sides after the ad-
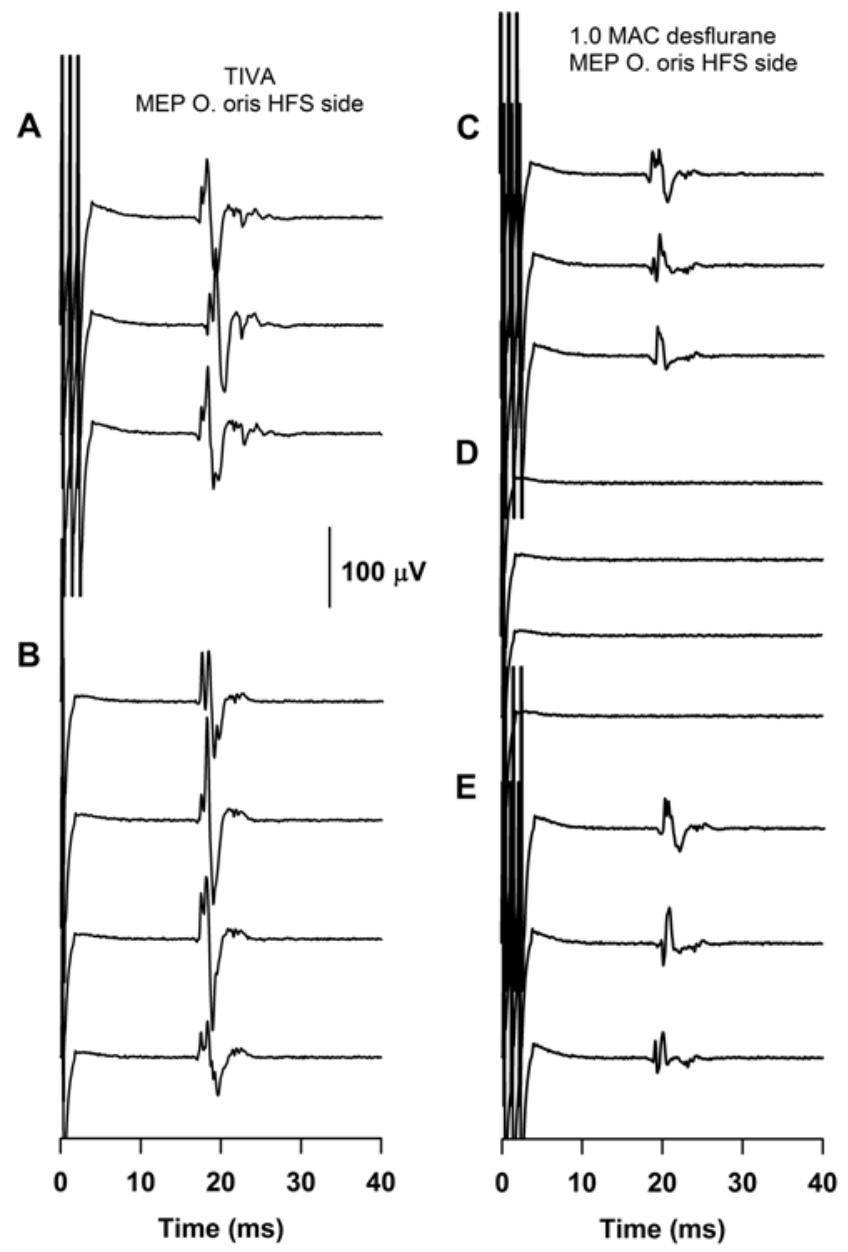

FIG. 1. Motor evoked potential recordings from the orbicularis oris $(\mathrm{O}$. oris) on the spasm side during MVD surgery for HFS. Patient MEPS were generated in response to multipulse stimulation $(\mathbf{A})$ or singlepulse transcranial electrical stimulation (B) after administration of TIVA. Desflurane (1.0 MAC) suppressed the MEP response when multipulse stimulation was used ( $\mathbf{C}$ and $\mathbf{E}$ ) and abolished the response to singlepulse stimulation (D).
TABLE 2. MEP latencies from combined facial muscle responses after single- or multipulse transcranial electrical stimulation

\begin{tabular}{lccc}
\hline \multirow{2}{*}{ Side } & \multicolumn{2}{c}{ MEP-Onset Latencies (mean \pm SEM) } & $p$ \\
\cline { 2 - 3 } & 1 Pulse, All Muscles & Multipulse, All Muscles & Value \\
\hline HFS $(\mathrm{msec})$ & $13.1 \pm 0.3$ & $14.6 \pm 0.5$ & $<0.03^{*}$ \\
\hline Control $(\mathrm{msec})$ & $13.6 \pm 1.0$ & $13.8 \pm 0.8$ & $0.86 \dagger$ \\
\hline $\mathrm{p}$ value & $0.57 \dagger$ & $0.86 \dagger$ & - \\
\hline
\end{tabular}

\footnotetext{
* According to Mann-Whitney rank-sum test.

$\dagger$ According to t-test.
}

ministration of TIVA. Table 1 details the MEP responses in patients after the administration of TIVA and desflurane according to individual facial muscle. We pooled these data to summarize the results (shown in Fig. 2). Desflurane predictably suppressed facial MEP amplitudes at both 0.5 and 1.0 MAC, but only 1.0-MAC desflurane produced statistically significant suppression regardless of the recorded side (Fig. 2; Table 1). Nevertheless, 1.0-MAC desflurane was significantly more potent in suppressing
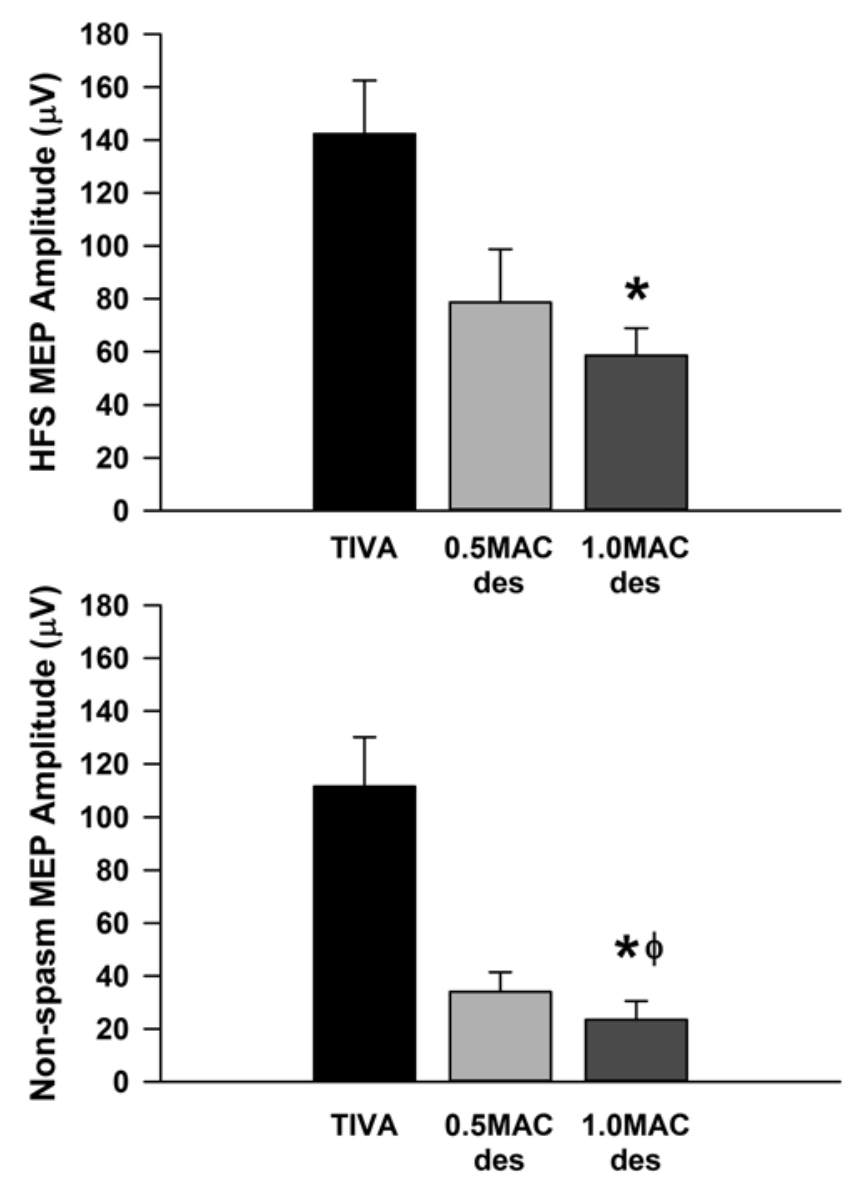

FIG. 2. Mean ( \pm SEM) MEP amplitudes (in microvolts) from pooled responses of the orbicularis oculi (HFS side only), orbicularis oris, and mentalis on the spasm (upper) and nonspasm (lower) sides of patients with HFS after the administration of TIVA or TIVA plus desflurane (des). ${ }^{*} p<0.01$ (1-way ANOVA with Holm-Sidak's method for multiple pairwise comparisons); $\phi p=0.03$ (Mann-Whitney rank-sum U-test, 1.0-MAC desflurane HFS vs control). 
facial MEPs on the control side than on the spasm side ( $79 \%$ vs $59 \%$, respectively; $p=0.03$, Mann-Whitney ranksum U-test).

To demonstrate that desflurane did not affect the peripheral facial nerve or neuromuscular junction, we examined the mentalis muscle $\mathrm{M}$ waves resulting from supramaximal mandibular branch stimulation. Data from 28 patients revealed an equal mean $\mathrm{M}$ wave amplitude of 1.8 $\pm 0.2 \mathrm{mV}$ after administration of TIVA or TIVA plus 1.0 MAC desflurane ( $\mathrm{p}=0.9$, paired t-test). The mean arterial blood pressure of patients who underwent TIVA was $77.0 \pm 2.8 \mathrm{~mm} \mathrm{Hg}$ when spasm-side MEPs were recorded versus $77.4 \pm 2.6 \mathrm{~mm} \mathrm{Hg}$ during control data collection ( $\mathrm{p}=0.8$, Mann-Whitney rank-sum U-test). Similarly, in patients who underwent TIVA plus desflurane, the mean arterial blood pressure was $75.9 \pm 2.1 \mathrm{~mm} \mathrm{Hg}$ during HFS MEP recordings and $75.8 \pm 2.2 \mathrm{~mm} \mathrm{Hg}$ during control-side MEP recordings ( $\mathrm{p}=1.0, \mathrm{t}$-test). Patient EEG status during the period of study fell into 1 of 2 descriptive categories: predominant alpha/delta or burst suppression. Analysis of the EEG data revealed no significant EEG differences in patients who underwent TIVA and those who underwent TIVA plus desflurane treatments, regardless of the side ( $\mathrm{p}$ $=1.0$, Fisher exact test).

\section{Discussion}

To our knowledge, this is the first study to contrast the effects of an inhalational anesthetic on facial MEPs in patients with HFS undergoing MVD surgery. It is notable that we found a suppressive effect of desflurane (1.0 MAC) on facial MEPs, but this effect was significantly less potent on the spasm side of the face. There was also a lower threshold stimulus needed to generate a facial MEP on the spasm side than on the nonspasm side. A greater excitability on the spasm side was also found with the observation that a single-pulse transcranial electrical stimulation was more likely to generate a facial MEP on the spasm side. The excitability differences of facial MEPs between the spasm and nonspasm sides and the differential effects of desflurane support the central hypothesis of pathophysiology in patients with HFS. Unlike the LSR, monitoring facial MEPs during MVD for HFS allows for bilateral recording and comparisons of facial motor excitability. This result may be of value in predicting the adequacy of surgical decompression and warrants further study. When monitoring facial MEPs during surgery, however, it remains important to recognize the inhibitory effects of inhalational anesthetics.

Inhalational anesthetics such as desflurane are well known for their suppressive effects on limb MEPs. ${ }^{2,3,27,31,34}$ The locus of action for MEP suppression is primarily at the level of the alpha motor neuron in the spinal cord..$^{16,19,28}$ The MEPs elicited by transcranial electrical stimulation are initiated from corticospinal axons; thus, the cortical actions of inhalational anesthetics do not contribute significantly to MEP suppression.,19 The same mechanisms apply to the corticobulbar tracts involved in facial MEP generation. In our study, the most likely locus of action for desflurane is the motor neurons of the facial nucleus, which would make desflurane a useful probe for investi- gating central versus peripheral mechanisms in patients with HFS. Our protocol enabled us to examine populations of facial motor neurons on both sides of the face, and we found differential effects of desflurane on spasm and nonspasm MEPs. Desflurane (1 MAC) significantly suppressed facial MEPs by 59\% on the spasm side and $79 \%$ on the control side. Although 0.5-MAC desflurane reduced mean MEP amplitudes, these reductions did not reach significance (regardless of the side), which is consistent with the observations of others. ${ }^{3,32}$ It is noteworthy that the degree of desflurane suppression on nonspasm facial MEPs was in the range of MEP suppression reported for limb muscles..$^{31,34}$

Differences in facial motor neuron excitability between the spasm and nonspasm sides are likely the key to understanding the differential effects of MEP suppression by desflurane. We believe that in patients with HFS, spasm-side facial motor neurons are more depolarized then those on the normal side. Thus, the depressive effects of desflurane are less potent, because the electrical stimulus to elicit an MEP need not depolarize the motor neuron membrane to the same degree as required on the less excitable nonspasm side. The result is a differential effect of desflurane on facial MEPs between sides. We also found that the success rates for generating facial MEPs were greater on the spasm side $(84 \%)$ than on the nonspasm side (74\%). Although it is tempting to speculate that this result also reflects evidence of enhanced excitability, it could well equally reflect the difference in the number of recording channels used for the spasm side (3 muscles) and for the nonspasm side ( 2 muscles). This difference might underscore the importance of recording from several facial muscles to obtain a broader picture of the facial motor neuron pool.

We observed no differences in EEG readings or mean arterial blood pressure of the patients during the periods of spasm data collection and those of nonspasm data collection. In addition, desflurane did not suppress the neuromuscular junction; mentalis muscle $\mathrm{M}$ waves were identical in patients who were given TIVA alone and in those who were given TIVA plus desflurane. Thus, we believe that differences in facial motor neuron excitability are responsible for the effects of desflurane that we observed in this study. We previously demonstrated significant MEP stimulus threshold differences between patients with HFS and patients who were undergoing posterior fossa procedures and concluded that patients with HFS exhibit elevated facial motor neuron excitability. ${ }^{36}$ However, we could not rule out that the comparison patients in that study had a hypofunctioning corticobulbar pathway when compared with the patients with HFS. Therefore, in the present study, we compared MEP stimulation thresholds on the spasm and nonspasm sides of patients with HFS during MVD surgery. The observed spasm-side MEP threshold of $162.9 \pm 10.1 \mathrm{~V}$ was similar to that found in our previous work, ${ }^{36}$ but it was significantly lower than that on the nonspasm side $(198.3 \pm 10.1 \mathrm{~V})$. This result confirmed that spasm-side facial motor neurons require less depolarizing stimuli to achieve a firing threshold. Similar results have been found when using transcranial magnetic stimulation in awake patients with HFS. ${ }^{14}$ 
Another method for assessing motor neuron excitability was conducted using single- and multipulse transcranial electrical stimulation to elicit facial MEPs. It is widely accepted that when a patient has been given general anesthesia, multipulse transcranial electrical stimulation is required to generate MEP responses..$^{15,19,26}$ In patients with HFS, however, single pulses are often sufficient to generate facial MEPs even after having been administered anesthesia. 6,36,37 We have interpreted this to indicate that in patients with HFS, the facial motor neurons are in an excitation state that requires little or no temporal summation for action potential initiation. Our results show that spasm-side MEPs were generated in $74 \%$ of the patients by using single-pulse transcranial stimulation but in only $31 \%$ of the patients on the nonspasm side. In a previous cohort, we also found that spasm-side facial MEPs were elicited with single-pulse stimulation in $76 \%$ of the patients with HFS. ${ }^{36} \mathrm{~A}$ criticism of the single-pulse method has been put forth from the belief that only multipulse stimuli can generate a true corticobulbar MEP. ${ }^{18}$ However, all singlepulse responses met corticobulbar MEP criteria, ${ }^{4}$ and we believe that these responses reflect a unique neurophysiological characteristic of patients with HFS that is absent in other patient groups. The value of studying facial MEPs in patients with HFS is that the generation of these responses occurs proximal to the neurovascular contact; therefore, differences in the MEP threshold or the number of stimulation pulses required to elicit a MEP response are established centrally, which supports the central hypothesis of pathophysiology in patients with HFS.

Dong et al. ${ }^{4}$ established the onset-latency criteria for corticobulbar-generated MEPs, and $10 \mathrm{msec}$ is considered the minimum acceptable value. Onset latencies of $<10$ msec are interpreted as extracranial activation of the facial nerve and thus not centrally generated. Using a single-pulse stimulation method, we found that MEP onset latencies met corticobulbar-generated criteria and were not different between the spasm- and nonspasm-side responses. However, we did observe longer mean onset latencies from spasmside MEPs elicited with multiple stimulation pulses (Table 1). We are unsure if this result is physiologically significant, because we previously found no differences in the onset latencies of MEPs generated with single-pulse stimuli and those generated with multipulse stimuli. ${ }^{36}$ In addition, an examination of the data revealed 3 responses from the multipulse group for which the onset latencies exceeded 20 msec, which is more than 2 standard deviations from the mean. Thus, the difference in onset latencies likely represents a sampling issue rather than a significant physiological phenomenon.

\section{Conclusions}

Motor neuron excitability and the actions of desflurane are both the result of physiological processes within the central nervous system. The suppressive effects of this inhaled anesthetic on facial MEPs were significantly less and facial motor neuron excitability was significantly greater on the spasm side than on the nonspasm side. These data provide new evidence that implicates a central pathophysiology unique to patients with HFS.
Volatile anesthetic agents should be used with caution if LSR or MEP responses are to be relied on during surgery, and no more than 0.5 MAC of desflurane should be incorporated into the anesthetic regimen. Recording from several facial muscles is also recommended. Although monitoring the LSR is commonly relied on for confirming the adequacy of decompression during surgery for HFS, facial MEPs also allow for bilateral recordings for comparisons of facial motor excitability that also may be of value for predicting the adequacy of surgical decompression and deserves further study.

\section{Acknowledgments}

We thank the members of the Department of Anesthesiology and Perioperative Medicine for their participation in this study. We also acknowledge K. Thompson, CNIM, and L. Vargas, CNIM, for their expert technical assistance.

\section{References}

1. Burke D, Bartley K, Woodforth IJ, Yakoubi A, Stephen JPH: The effects of a volatile anaesthetic on the excitability of human corticospinal axons. Brain 123:992-1000, 2000

2. Chen Z: The effects of isoflurane and propofol on intraoperative neurophysiological monitoring during spinal surgery. J Clin Monit Comput 18:303-308, 2004

3. Chong CT, Manninen P, Sivanaser V, Subramanyam R, Lu N, Venkatraghavan L: Direct comparison of the effect of desflurane and sevoflurane on intraoperative motor-evoked potentials monitoring. J Neurosurg Anesthesiol 26:306-312, 2014

4. Dong CC, Macdonald DB, Akagami R, Westerberg B, Alkhani A, Kanaan I, et al: Intraoperative facial motor evoked potential monitoring with transcranial electrical stimulation during skull base surgery. Clin Neurophysiol 116:588-596, 2005

5. Eekhof JLA, Aramideh M, Speelman JD, Devriese PP, Ongerboer De Visser BW: Blink reflexes and lateral spreading in patients with synkinesia after Bell's palsy and in hemifacial spasm. Eur Neurol 43:141-146, 2000

6. Fernández-Conejero I, Ulkatan S, Sen C, Deletis V: Intra-operative neurophysiology during microvascular decompression for hemifacial spasm. Clin Neurophysiol 123:78-83, 2012

7. Fukuda M, Oishi M, Hiraishi T, Fujii Y: Facial nerve motorevoked potential monitoring during microvascular decompression for hemifacial spasm. J Neurol Neurosurg Psychiatry 81:519-523, 2010

8. Fukuda M, Oishi M, Takao T, Hiraishi T, Sato Y, Fujii Y: Monitoring of abnormal muscle response and facial motor evoked potential during microvascular decompression for hemifacial spasm. Surg Neurol Int 3:118, 2012

9. House JW, Brackmann DE: Facial nerve grading system. Otolaryngol Head Neck Surg 93:146-147, 1985

10. Ishikawa M, Namiki J, Takase M, Ohira T, Nakamura A, Toya S: Effect of repetitive stimulation on lateral spreads and F-waves in hemifacial spasm. J Neurol Sci 142:99-106, 1996

11. Jannetta PJ, Abbasy M, Maroon JC, Ramos FM, Albin MS: Etiology and definitive microsurgical treatment of hemifacial spasm. Operative techniques and results in 47 patients. J Neurosurg 47:321-328, 1977

12. Kang MC, Choi YS, Choi HK, Lee SH, Ghang CG, Kim $\mathrm{CH}$ : Efficacy of the disappearance of lateral spread response before and after microvascular decompression for predicting the long-term results of hemifacial spasm over two years. J Korean Neurosurg Soc 52:372-376, 2012

13. Kaufmann AM, Wilkinson MF: Microvascular decompression for hemifacial spasm, in Schmidek HH, Roberts DW 
(eds): Schmidek \& Sweet Operative Neurosurgical Techniques: Indications, Methods, and Results, ed 5. Philadelphia: Saunders, 2006, pp 1473-1490

14. Kojima A, Ohira T, Takase M, Kawase T: Long-latency response to transcranial magnetic stimulation in patients with hemifacial spasm. Electroencephalogr Clin Neurophysiol 109:285-289, 1998

15. Legatt AD, Emerson RG: Motor evoked potential monitoring-it's about time. J Clin Neurophysiol 19:383-386, 2002

16. Loughnan BA, Anderson SK, Hetreed MA, Weston PF, Boyd SG, Hall GM: Effects of halothane on motor evoked potential recorded in the extradural space. Br J Anaesth 63:561-564, 1989

17. Lu AY, Yeung JT, Gerrard JL, Michaelides EM, Sekula RF Jr, Bulsara KR: Hemifacial spasm and neurovascular compression. ScientificWorldJournal 2014:349319, 2014

18. MacDonald DB: Intraoperative facial motor evoked potentials? J Neurosurg 106:517-519, 2007

19. MacDonald DB, Skinner S, Shils J, Yingling C: Intraoperative motor evoked potential monitoring - a position statement by the American Society of Neurophysiological Monitoring. Clin Neurophysiol 124:2291-2316, 2013

20. Møller AR: Vascular compression of cranial nerves. I. History of the microvascular decompression operation. Neurol Res 20:727-731, 1998

21. Møller AR, Jannetta PJ: Microvascular decompression in hemifacial spasm: intraoperative electrophysiological observations. Neurosurgery 16:612-618, 1985

22. Møller AR, Jannetta PJ: Monitoring facial EMG responses during microvascular decompression operations for hemifacial spasm. J Neurosurg 66:681-685, 1987

23. Møller AR, Jannetta PJ: Physiological abnormalities in hemifacial spasm studied during microvascular decompression operations. Exp Neurol 93:584-600, 1986

24. Nielsen VK: Pathophysiology of hemifacial spasm: I. Ephaptic transmission and ectopic excitation. Neurology 34:418426, 1984

25. Nielsen VK: Pathophysiology of hemifacial spasm: II. Lateral spread of the supraorbital nerve reflex. Neurology 34:427-431, 1984

26. Pechstein U, Nadstawek J, Zentner J, Schramm J: Isoflurane plus nitrous oxide versus propofol for recording of motor evoked potentials after high frequency repetitive electrical stimulation. Electroencephalogr Clin Neurophysiol 108:175-181, 1998

27. Pelosi L, Stevenson M, Hobbs GJ, Jardine A, Webb JK: Intraoperative motor evoked potentials to transcranial electrical stimulation during two anaesthetic regimens. Clin Neurophysiol 112:1076-1087, 2001

28. Péréon Y, Bernard JM, Nguyen The Tich S, Genet R, Petitfaux F, Guihéneuc P: The effects of desflurane on the nervous system: from spinal cord to muscles. Anesth Analg 89:490495, 1999

29. Poignonec S, Vidailhet M, Lamas G, Fligny I, Soudant J, Jedynak P, et al: Electrophysiological evidence for central hyperexcitability of facial motoneurons in hemifacial spasm. Eur Arch Otorhinolaryngol 1994:S216-S217, 1994

30. Saklad M: Grading of patients for surgical procedures. Anesthesiology 2:281-284, 1941
31. Sekimoto K, Nishikawa K, Ishizeki J, Kubo K, Saito S, Goto F: The effects of volatile anesthetics on intraoperative monitoring of myogenic motor-evoked potentials to transcranial electrical stimulation and on partial neuromuscular blockade during propofol/fentanyl/nitrous oxide anesthesia in humans. J Neurosurg Anesthesiol 18:106-111, 2006

32. Sloan TB, Toleikis JR, Toleikis SC, Koht A: Intraoperative neurophysiological monitoring during spine surgery with total intravenous anesthesia or balanced anesthesia with $3 \%$ desflurane. J Clin Monit Comput 29:77-85, 2015

33. Tankéré F, Maisonobe T, Lamas G, Soudant J, Bouche P, Fournier E, et al: Electrophysiological determination of the site involved in generating abnormal muscle responses in hemifacial spasm. Muscle Nerve 21:1013-1018, 1998

34. Ubags LH, Kalkman CJ, Been HD: Influence of isoflurane on myogenic motor evoked potentials to single and multiple transcranial stimuli during nitrous oxide/opioid anesthesia. Neurosurgery 43:90-95, 1998

35. Wilkinson MF, Chowdhury T, Mutch WAC, Kaufmann AM: Is hemifacial spasm a phenomenon of the central nervous system? The role of desflurane on the lateral spread response. Clin Neurophysiol 126:1354-1359, 2015

36. Wilkinson MF, Kaufmann AM: Facial motor neuron excitability in hemifacial spasm: a facial MEP study. Can J Neurol Sci 41:239-245, 2014

37. Wilkinson MF, Kaufmann AM: Monitoring of facial muscle motor evoked potentials during microvascular decompression for hemifacial spasm: evidence of changes in motor neuron excitability. J Neurosurg 103:64-69, 2005

38. Xia L, Zhong J, Zhu J, Dou NN, Liu MX, Li ST: Delayed relief of hemifacial spasm after microvascular decompression. J Craniofac Surg 26:408-410, 2015

39. Yamashita S, Kawaguchi T, Fukuda M, Suzuki K, Watanabe M, Tanaka R, et al: Lateral spread response elicited by double stimulation in patients with hemifacial spasm. Muscle Nerve 25:845-849, 2002

\section{Disclosures}

The authors report no conflict of interest concerning the materials or methods used in this study or the findings specified in this paper.

\section{Author Contributions}

Conception and design: Wilkinson, Chowdhury, Mutch. Acquisition of data: Wilkinson. Analysis and interpretation of data: Wilkinson. Drafting the article: all authors. Critically revising the article: all authors. Reviewed submitted version of manuscript: all authors. Approved the final version of the manuscript on behalf of all authors: Kaufmann. Statistical analysis: Wilkinson. Administrative/technical/material support: Mutch. Study supervision: Kaufmann, Wilkinson.

\section{Correspondence}

Anthony M. Kaufmann, GB-137 Health Science Centre, 820 Sherbrook St., Winnipeg, MB R3A 1R9, Canada. email: akaufmann@hsc.mb.ca. 